\title{
Efficacy of Artemisinin-lumefantrine for the treatment of uncomplicated malaria after more than a decade after its use in Kenya
}

gabriel m kishoyian ( $\sim$ gkishoyian@gmail.com )

Kenya Medical Training College

Eliud N.M. Njagi

Kenyatta University

George 0. Orinda

Kenyatta University

Francis T. Kimani

kenya medical research institute

Kevin Thiongo

Kenya Medical Research Institute

Damaris Matoke-Muhia

Kenya Medical Research Institute

Research

Keywords: Efficacy, Artemether-lumefantrine, falciparum malaria, Kenya.

Posted Date: June 29th, 2020

DOl: https://doi.org/10.21203/rs.3.rs-22339/v2

License: (c) (i) This work is licensed under a Creative Commons Attribution 4.0 International License.

Read Full License 


\section{Abstract}

Background: Plasmodium falciparum resistance to antimalarial drugs remains to be a major threat to the control of the disease globally. After the deployment of artemisinin-based combination therapy (ACT), there have been reports of reduced sensitivity of the drug to parasite clearance. In Kenya, artemisininlumefantrine $(\mathrm{AL})$ is the recommended first-line drug in the treatment of uncomplicated malaria. This study sought to assess the efficacy of AL after its reintroduction in Kenya, a decade later. We assessed clinical and parasitological responses of children under five years in May and November 2015 in Chulaimbo sub-County, Kisumu, Kenya.

Method: Patients of $\geq 6$ and $\leq 60$ months of age with confirmed Plasmodium falciparum mono-infection were enrolled in the study. The children were inpatient for close monitoring, they were treated with a standard dose of AL under supervision of a qualified nurse and followed up for 28 days. We monitored treatment adherence and responses. Efficacy of artemether lumefantrine on Plasmodium falciparum was determined.

Results: Of the 90 patients enrolled, fourteen (14) were lost to follow-up, with 76 completing the study period. Seventy-five patients $75(98.7 \%)$ cleared the parasitemia within 48 hours while one $(1.3 \%)$ cleared on day 3 . There was $100 \%$ clinical and parasitological parasite clearance. Conclusion: Artemisinin lumefantrine was found to be highly efficacious to plasmodium falciparum parasites in children aged $\geq 6$ and $\leq 60$ months. The results reported here indicate that the drug can be used to treat uncomplicated malaria in the study population. However, there is need for continued monitoring of its effectiveness in children and adults to counter the threat of resistance.

\section{Introduction}

In spite of the tremendous decline of malaria burden over the past decade, the disease still remains a major public health concern globally with sub-Saharan Africa bearing the greatest burden [1]. In 2018, approximately 228 million malaria cases and 405,000 deaths were reported worldwide. Of this $92 \%$ of cases and $93 \%$ of deaths were from sub-Saharan Africa, with children under five years of age and expectant mothers bearing the highest burden [1]. There are 5 species of malaria parasites that infect humans: Plasmodium .falciparum, $P$. ovale, $P$. malaria, $P$. vivax and $P$. knowlesi with $P$. falciparum being the most life-threatening, and responsible for the majority of morbidity and mortality [1]. World Health Organization Global Malaria Programme (WHO/GMP) recommends three primary malaria interventions comprising of; 1) Prompt diagnosis \& treatment with effective medicines; 2) Insecticide-treated nets (ITNs) distribution to achieve full coverage of populations at risk of malaria; and 3) Indoor Residual Spraying (IRS) as a major means to reduce and eliminate malaria transmission [2, 3]. Since the initiation of the Roll Back Malaria (RBM) Project, over a decade ago there has been increased Long Lasting Insecticide Treated Nets (LLINs) coverage and intense case management in most countries where malaria is endemic and success has been reported due to the decline in malaria transmission [4]. 
Despite the success of the current malaria control tools, more stringent measures of monitoring efficiency and novel disease interventions are needed. Integration of preventive chemotherapy and case treatment into mainstream malaria interventions is of importance [5]. Early case detection and prompt treatment is key in minimizing malaria-related morbidity and mortality with appropriate use of antimalarial drugs remaining the cornerstone of malaria control [1]. However, it's being threatened by resistance of parasites to anti-malarial drugs that have been reported to occur in Southeast Asia with potential spread to Africa. In the early 1940 s, chloroquine (CQ) was the drug of choice for the treatment of malaria in many countries having been confirmed as an anti-malarial drug with a quick metabolism, good curative effect including affordable cost [6]. The first case of Plasmodium falciparum resistance to CQ was reported in Thai-Cambodia border in Southeast Asia and South America in the 1950s and thereafter spread to all malaria-endemic regions globally, with emergence in Africa in the 1970s [6]. In Africa, resistance to CQ led to its withdrawal as an antimalarial drug and replaced with sulfadoxine-pyrimethamine (SP) in the early 1980s [7]. Malawi was the first country in the continent to cease the administration of CQ in malaria chemotherapy in the year 1993 [8]. In Tanzania, CQ was used as a first-line malaria treatment drug since the 1970s but due to high levels of resistance, it was replaced with SP in the year 2001. This was shortlived, as resistance emerged soon after, thus necessitating the adoption of AL in 2006 [8]. The World Health Organization recommends that malaria-endemic countries should monitor the efficacy of nationally recommended artemisinin-based combination therapy (ACT) in order to make decisions on treatment options [11].

Assessment of efficacy of antimalarial drugs include methods such as: (1) In vivo clinical trials - this involves treatment and patients followed for 28 or 42 days as recommended by WHO and the outcome classified as early treatment failure (ETF), late clinical failure (LCF), late parasitological failure (LPF) and adequate clinical and parasitological response (ACPR) [11], (2) In vitro assays which are achieved by measuring the susceptibility of malaria parasites in a culture uses fresh parasites isolates and usually measures the drug concentration in which $50 \%$ of the parasite growth is inhibited (IC50) compared to the unexposed control [12]. Resistance to artemisinin is monitored using ring-stage survival assay (RSA), which measures the proportion of viable parasites that develop into second-generation rings or trophozoites after a six-hour pulse of dihydroartemisinin [13].

In Kenya, CQ resistant Plasmodium falciparum was reported in 1977, whereby by 1998 resistant levels had reached 70\% [6]. Like other sub-Saharan countries, Kenya replaced CQ with SP in 1999 as the official first-line in the treatment of uncomplicated malaria [14]. As a result of widespread increasing reports of SP efficacy being compromised in Kenya especially at the coastal region [15-16], prompted another policy change in the treatment of malaria in 2004 by introducing the use of Coartem ${ }^{\mathrm{TM}}$, an artemetherlumefantrine and thereafter was made available in government hospitals in 2006 [8]. However, as with other drugs, the curative effect of ACT has declined gradually with studies showing slow parasites clearance to Plasmodium falciparum positive patients over time in Southeast Asia [17]. Due to the threat of potential artemisinin drug resistance, WHO recommends regular surveillance to monitor the performance of antimalarial drugs in malaria-endemic countries [2]. This study was carried out with the 
primary objective of assessing the clinical efficacy of AL with a six-dose regiment treatment of uncomplicated Plasmodium falciparum malaria using the WHO therapeutic efficacy protocols after its introduction in Kenya over a decade.

\section{Materials And Methods}

\section{Study area}

We conducted the trial in Chulaimbo sub County Hospital, Lake Region of Kisumu County, Western Kenya. The area altitude is $1,131 \mathrm{M}$ above sea level with yearly rainfall between $1200 \mathrm{~mm}$ and $1300 \mathrm{~mm}$. The humidity ranges between $50 \%-68 \%$ with temperatures ranging between $20^{\circ} \mathrm{C}$ and $35^{\circ} \mathrm{C}$. Chulaimbo Sub County is a malaria-endemic zone with stable Plasmodium falciparum transmission [18]. Malaria infection accounts for $38 \%$ of all outpatient hospital visits and $40 \%$ of all admissions being children below five years and expectant mothers [6].

\section{Study population}

Children aged $\geq 6$ and $\leq 60$ months visiting the outpatient clinic of Chulaimbo Sub County Hospital with signs of uncomplicated malaria were recruited for the study in the month of May 2015 to November 2015. Inclusion criteria was children who were residents of the area, with a body weight of $\geq 5 \mathrm{~kg}$, history of fever in the previous 24 hours or fever with temperature $\geq 37.5^{\circ} \mathrm{C}$, infected only with Plasmodium falciparum and parasitemia in the range of 2,000 to 200,000 asexual parasites per microliter of blood, no general danger signs of severe and complicated malaria (prostration, breathing difficulties, severe anemia, convulsions and inability to drink or vomiting). Written consent was obtained from the parent or guardian of the child before recruitment into the study. Patients were excluded from the study if they were below 6 months and above 60 months, body weight less than $5 \mathrm{~kg}$ with history of fever for more than 24 hours with a temperature of above $37.5^{\circ} \mathrm{C}$, multiple infections apart from malaria. Also, the inability to take the drug orally, having taken antimalarial chemotherapy in the past two weeks, evidence of liver disease or acute infection other than malaria and unwilling to participate were excluded from the study.

\section{Sampling design}

- This was a single-arm prospective in vivo study intended to assess the efficacy of

- Artemether-lumefantrine resistant after treatment of uncomplicated malaria using WHO

- guidelines

\section{Sample size determination}

The sample size determination was calculated using the formula $n=Z^{2} p(1-P) / e^{2}$ as described by Lwanga and Lemeshow [19].

Where; 
$Z$ = standard normal deviation of the required confidence.

$\mathrm{n}=$ the desired sample size

$\mathrm{p}=$ Proportion in the target population estimated to have characteristic being measured. According to KIAS, 2010 the prevalence of malaria stands at $40 \%$ or 0.4 .

$\mathrm{e}=$ the level of statistical significance set.

Therefore substituting $\mathrm{Z}$ for $1.96,0.38$ for $\mathrm{P}$ and 0.1 for $\mathrm{e}$, then the minimum sample size was

$1.96^{2} \times 0.38(1-0.38) / 0.1^{2}=90$.

\section{Sample collection}

Approximately, $0.05 \mathrm{ml}$ of blood from a finger prick was collected; thick and thin smears were prepared on two different slides. One of the slides was stained with 10\% Giemsa for 10-15 min and examined by microscopy to detect the presence of the malaria parasites and density estimated. The second thin film blood slide was stained with 3\% Giemsa for 30-45 min and used to determine the species and presence of gametocytes. Parasitemia was measured by counting the number of asexual parasites against 200 leucocytes in thick blood films. Parasite density per $\mu \mathrm{L}$ of blood calculated by multiplying the total count by 40 , assuming that $1 \mu \mathrm{L}$ of blood had a mean count of 8000 leucocytes [20]. The blood slides were declared negative when the examination of 100 high power fields did not show the existence of any malaria parasite. For quality control, each slide was re-examined by a second laboratory technologist, and those with discrepant results were re-examined by a third technologist. Final parasitemia was calculated from the average between the two readings [20].

\section{Treatment, clinical monitoring and follow-up}

Treatment with AL was done for 3 days according to WHO weight-based regime [10]. A fixed-dose combination of $20 \mathrm{mg}$ of artemether and $120 \mathrm{mg}$ lumefantrine per tablet, translating to one, two or three tablets per patient depending on the individual weight respectively was administered. A full course of $A L$ consisted of 6 doses given twice daily (8 hours apart on day 0 and 12-hour on days 1 and 2) with no gametocide drug added. All drugs used for this study were provided by the Ministry of Health. Patients were observed for 20 minutes to make sure they did not vomit. If vomiting occurred, a repeat dose was given after the patient was in a state of swallowing. Any patient who persistently vomited was withdrawn and treated with parenteral artesunate or quinine according to the national guidelines for the management of severe malaria [21]. Besides, paracetamol was given to all patients with body temperature $\geq 38^{\circ} \mathrm{C}$. Patients were admitted at the health facilities for close monitoring, the drugs were administered orally at the health facility under direct observation of a nurses throughout the three days. The drug was crushed and mixed with spoonful of porridge and little sugar to minimize vomiting and was given to each child to gulp. No serious adverse effects was observed throughout the 28 days follow up. 
On completion of the dose, after three days, the patients were allowed to go home and follow-up visits were done on day 7, 14, 21 and 28 or at any time the patient felt unwell. Parents/guardians were informed and encouraged to bring their children back to the clinic whenever they were unwell without waiting for scheduled visits. Parents who did not show up during their scheduled visit by mid-day of the same day they were visited at home by a member of a study team and asked to come to the health facility. If a patient could not be traced for scheduled follow-up, he/she was classified as lost to follow-up. During the visits, both clinical and parasitological assessment were performed. Patients with recurrent infections occurring on day 7 and afterward were treated with artesunate or quinine (tablets, injection/intravenous) based on clinical presentation according to WHO protocol [10].

\section{Treatment outcome classification}

The clinical outcome was corrected with the parasitological cure on day 28 based on WHO in-vivo drug trial protocol of 2009 [10]. Treatment outcome was classified as either early treatment failure (ETF) which refer to danger signs for severe malaria on day 1,2 or 3 , in the presence of parasitemia; parasitemia on day 2 being higher than on day 0 count irrespective of axillary temperature; parasitemia on day 3 with axillary temperature $\geq 37.5^{\circ} \mathrm{C}$; parasitemia on day $3 \geq 25 \%$ of count on day 0 . Late clinical failure (LCF) which include the development of danger signs for severe malaria after day 3 in the presence of parasitemia, without previously meeting any of the criteria of ETF; presence of parasitemia and axillary temperature $\geq 37.5^{\circ} \mathrm{C}$ or history of fever on any day from day 4 to day 28 , without previously meeting any of the criteria of ETF. Late parasitological failure (LPF). This refers to the presence of parasitemia on any day from day 7 to day 28 and axillary temperature $<37.5^{\circ} \mathrm{C}$, without previously meeting any of the criteria of early treatment failure or late clinical failure. Adequate clinical and parasitological response (ACPR). This is the absence of parasitemia on day 28 irrespective of axillary temperature without previously meeting any of the criteria of ETF, LTF or LPF.

\section{DNA extraction using chelex method}

The DNA extraction from dried blood spots (DBS) was done as described by Walsh et al. [22] while extraction from DBS as explained by Warhurst et al. [23] using QIAamp DNA Mini kit (QIAGEN Inc., Germany) according to the manufacturer's instructions. Briefly, each dried filter paper was cut into small pieces and soaked in Saponin-phosphate buffered saline (PBS) overnight at $4^{\circ} \mathrm{C}$. This was followed by washing with $1 \times$ PBS and incubated for 30 minutes. The brown solution from the tube was discarded and 50 microliters of the stock $20 \%$ solution and 150 microliters of DNAse free water were added followed by vigorous vortexing. The tubes were then heated at $100^{\circ} \mathrm{C}$ and centrifuged at $10,000 \mathrm{~g}$ for two minutes. Lastly, the supernatant was transferred to a new tube, spun again, a final transfer done and the DNA stored at $-20^{\circ} \mathrm{C}$.

\section{Merozoites surface antigens screening}


During the study, patients with recurrent parasitemia on day 7 onwards, a sample was collected on filter paper. Genotypes from day 0 and day of recurrent parasites was compared to differentiate recrudescence from the new infection. However, there was no re-infection from the follow up of the children who completed the study up to day 28.

\section{Amplification of Pf18sRNA gene}

In order to confirm Plasmodium falciparum species infections, amplification of 18s RNA gene was done. Primers pairs designed by [24] of 18R-18F were used; (5'-CTGAGTCGAATGAACTAGCT-3') and (5'CCATTTTACTCGCAATAACG-3') respectively. The PCR reaction included $\mathrm{MgCl}_{2}, 400 \mathrm{nM}, 200 \mathrm{nM}$ of primers, $1 \mathrm{U}$ of Taq Polymerase and $1 \mu \mathrm{L}$ of DNA template. Amplification was done using PCR that included: the initial denaturation at $94^{\circ} \mathrm{C}$ for 3 minutes, followed by 30 cycles of denaturation at $94^{\circ} \mathrm{C}$ for 1 minute, annealing at $55^{\circ} \mathrm{C}$ for 2 minutes and extension at $72^{\circ} \mathrm{C}$ for 2 minutes, and the final extension of $72^{\circ} \mathrm{C}$ for 10 minutes the reaction was held at $4^{\circ} \mathrm{C}$.

\section{Results}

\section{Demographic characteristics}

A total of 151 children were screened between April and November 2015 for possible participation in the study. Of these, 26 had no malaria, 19 declined to participate, 2 had mixed infection while 14 had malaria with other complications. However, the 90 respondents satisfied the criteria for inclusion in the study as shown in Figure 1. Out of the 90 subjects, 14 (15.6\%) were lost (four, two, five and three) and were excluded on day 7, 14, 21 and 28 during follow-up leaving 76 (84.4\%) P. falciparum positive children that completed the study-up to day 28 as shown in Figure 2.

The clinical characteristics exhibited by the children included hotness of the body, headache, chills, nausea, vomiting and general malaise at day 0 . Also, there was no gametocyte detected among the children from day 0 to day 28 . The median age for males was 30 months while the range was $12-56$ months. The median age for females was 30 months while the range was $15-58$ months. The weight mean in $\mathrm{Kg}$ and the standard deviation was 14.07 and \pm 2.970 respectively. The minimum and maximum age were 12 and 58 months, while weight was $8 \mathrm{~kg}$ and $20 \mathrm{~kg}$ respectively. According to sex, we had 43 $(56.6 \%)$ males and $33(43.4 \%)$ females. The geometric mean of microscopic parasite count on day 0 (before treatment) was 120,595 parasites/ $\mu \mathrm{L}$ while the standard deviation was $163,395.1$ (95\% Cl: 82319.4-15887.89). On day 1 , the parasite count had a mean and standard deviation of 3508.93 and 11,783 respectively ( $95 \% \mathrm{Cl}$ : 797.8-6220.7), on day 2 , the parasite count had a mean and standard deviation of 24 and 170.9 (95\% Cl: 15.0-63.3) (Table 1).

\section{Table 1: Profile of children on therapeutic in the study population}




\begin{tabular}{cc}
\hline Characteristics & Artemether-lumefantrine \\
\hline Memale n (\%) & $\mathrm{n}=76$ \\
Median age in months (Males) & $43(56.6 \%)$ \\
Age range in months (Males) & $33(43.4 \%)$ \\
Median age in months (Females) & 30 \\
Age range in months (Females) & $(12-56)$ \\
Temperature mean on day 0 & 30 \\
Temperature on day 3 & $(15-58)$ \\
Parasitemia (per $\mu \mathrm{L}$ ) on day 0 geometric mean and SD respectively & $120,595.0$ and $163,395.1$ \\
Range & $880-832,000$ \\
\hline
\end{tabular}

\section{Therapeutic efficacy outcomes}

Of the 90 patients who participated in the study as shown in Table 2, 14 (15.6\%) were lost (four, two, five and three) and were excluded on day 7, 14, 21 and 28 leaving 76 (84.4\%) completing the follow-up to day 28. Within 48 hours (2days) of treatment, 75 (98.7\%) had cleared parasitemia and 1 (1.3\%) had parasitemia but cleared on day 3 as shown in Figure 3 with no recrudescent infection observed in the study during the follow up to day 28 . The mean body temperature on day 0 was $38.12 \pm$ while on day 3 it subsided to $36.5 \pm 0.62$ (Figure 4). However, on day 1 of treatment 58 (76.3\%) (95\% Cl 36.7-37.1) of the children were febrile while the afebrile were 18 (23.7\%) (37.5-38.7). On day 2, the temperature had returned to normal (afebrile) for all the respondents. In addition, there was no serious adverse effect during the follow up and no child that vomited. Also there were no discordance between the microscopy reading hence there was no need to add a third reader. PCR correction could not be done since there was no re-infection during the follow up to day 28 hence performing PCR-corrected (genotyped-correct) would be the same.

Though this kind of study requires survival curve analysis as shown in Figure 3, there were no therapeutic failures since the results showed 100\% ACPR. The presence of therapeutic failures allows for the analysis of molecular markers associated with artemether lumefanrine such as Pfmdr1 gene and K13 propeller gene. In addition, after the amplification of the Pf18sRNA, the results confirmed all the samples were those of Plasmodium falciparum species after detecting the highly conserved region (18s RNA) of the species-specific plasmodium falciparum.

\section{Table 2: Therapeutic efficacy of AL}




\begin{tabular}{cc}
\hline Variables & Frequency \\
\hline Parasitemia on day 3 & $0(0.0 \%)$ \\
ETF & $0(0.0 \%)$ \\
LCF & $0(0.0 \%)$ \\
LPF & $0(0.0 \%)$ \\
ACPR & $76(100 \%)$ \\
Initial no. of samples & 90 \\
Lost to follow up on day 7 & 4 \\
Lost to follow up on day 14 & 2 \\
Lost to follow up on day 21 & 5 \\
Lost to follow up on day 28 & 3 \\
Total baseline & 76 \\
\end{tabular}

\section{Discussion}

The instant and extensive increase of anti-malarial drug resistance are hindering the progress of malaria control [25-26]. In this study, we confirm a high cure rate and efficacy (100\% ACPR) of the nationally recommended $\mathrm{AL}$ for the treatment of uncomplicated malaria since its introduction in Kenya over a decade ago. The outcome of these study showed that there was no re-infection during the 28 day follow up hence no need for PCR correction since PCR-corrected (genotyped-correct) would be the same. Also, the clearance of parasitemia was attained on day 3 an indicative of an extraordinary parasite clearance rate. However, though the $100 \%$ adequate clinical and parasitological response was observed in the present study, the results were comparable with studies in Papua New Guinea which confirms a high efficacy rate 97.8\% [27]. These studies agree with the observation in Ethiopia, which shows a high therapeutic cure rate of above $98 \%$ [5]. The high cure rate of AL, especially in children less than five years is a good sign since treatment failure manifest easily in this age group because of low immunity [28].

In this study, AL has shown high efficacy similar to one carried in Tanzania and other parts of Africa primarily supporting the high efficacy rates of AL despite its use in the continent for over ten years [2930]. The elevated parasite clearance rates may possibly be described by the steadfast act of artemether to clear parasites biomass leading to a quick resolution of clinical manifestation. An indication of suspected Artemisinin resistance according to the WHO is the presence of delayed parasite clearance showing a slope half-life $>5$ hours or day 3 positive rate $<10 \%$ [11]. In the current study, immediate clearance of parasites after AL dose indicate the absence of Artemisinin resistance. This outcome correlate to a number of studies undertaken earlier in Kenya, Uganda, Somalia, Mali, and several other African countries indicating that Artemisinin resistance has not appeared in the African continent [29, 31]. The component drug, lumefantrine, is a gradually acting drug with a long half-life time ranging from 4-7 days [32]. This leads to successive accumulation of the drug after the completion of the full dose sufficient enough for the elimination of residual parasites and possibly prevention of new infection [32]. 
Among the Artemisinin-based combination therapies, artesunate-amodiaquine (ASAQ), dihydroartemisinin-piperaquine (DHA/PPQ) and AL are currently recommended ACTs for the treatment of uncomplicated falciparum malaria in malaria endemic regions globally [11]. Several recent studies have observed that these ACTs have maintained high efficacy (cure rate $\geq 95 \%$ ) in many of these countries, despite their use for more than a decade [28]. Nevertheless, a study carried out in Angola from two different regions in 2013 and 2015 showed a lower efficacy (<90\% cure rate) of AL. In these studies, the administration of the evening dose was not supervised hence no confirmation that this dose was consumed by the patient. It also meant that the lower cure rate observed in the two regions of Angola in those two years was contributed as a result of a sub-therapeutic doses of the AL or might signal reduced efficacy [28]. The efficacious reported here for AL was also observed in northwest Ethiopia where there was the absence of ETF, confirming nonexistence of possible Artemisinin-resistant Plasmodium falciparum in the study area. Despite the study showing the absence of ETF with low recurrent malaria (1 LTF), the outcome of the Ethiopian study point to a highly therapeutic efficacy of both partners of AL [33].

Factors such as host immunity, nutritional, initial parasitemia level, pharmacokinetics, and pharmacodynamics may influence the therapeutic efficacy of a drug apart from inherent parasite susceptibility [33]. Any of the above may contribute to low efficacy of a highly efficacious drug. At the same time, resistant parasites may be cleared with the help of the immune system resulting in exaggerated efficacy of otherwise a less efficacious antimalarial drug [33]. The unfortunate emergence and potential spread of Artemisinin-resistance parasites in Southeast Asia and changes in sensitivities to Artemisinin partner drugs have raised concern globally [34].

For example, in another clinical efficacy studies in Ethiopia observed that among the study population, there were five treatment failures, $1(1.1 \%)$ LTF and 4(4.5\%) were LPF while 84 (94.4\%) ACPR confirming that the treatment of uncomplicated malaria using $\mathrm{AL}$ has a high clearance rate similar to the current study which has observed a 100\%.ACPR. These studies have shown high efficacy of AL in the treatment of uncomplicated malaria and agree with findings from other east African countries and more important its rapid elimination sexual stages of Plasmodium falciparum and the activity of the partner drug on the

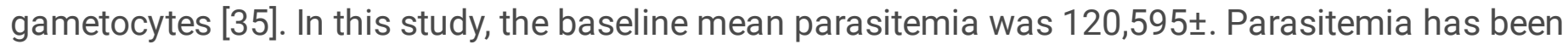
linked to the degree of malaria severity and hence it's an important parameter to help in the decision of the type of treatment to be initiated. It is also an epidemiological implication parameter as it indicates the level of transmission in a specific area. The level of malaria endemicity in the current study is characterized as holoendemic. According to the health facility's official data, malaria incidence in the area is seasonal, subject to the amount and length of rainfall.

However, in the current study, no gametocytes were detected from day 0 to day 28 . A study in Nigeria observed that gametocytes carriage reduction after treatment with AL in children with uncomplicated Plasmodium falciparum malaria. Also, a similar study in Thailand showed that when artemisisnin derivatives were introduce as component of the first-line treatment, there was tremendous reduction in the incidence of clinical Plasmodium falciparum [35]. Studies have observed that ACT has been associated with decline in transmission of malaria, which is due to the rapid elimination of asexual parasites, and 
partly due to their effects on gametocytes [36]. Gametocytes clearance after treatment reduces malaria transmission and prevent the selection including the spread of resistance malaria parasites. Nevertheless, ACT is highly effective against asexual stages and immature gametocytes [36]. However, ACT is known by its rapid parasite clearance more rapidly than other antimalarial such as quinine. In addition the drug target not only the late erythrocytic stage but also early stages of the parasites [37]. This shows the potent antimalarial property of Coartem ${ }^{\circledR}$ targeting the blood stage parasite.

We observed that fever was associated with discomfort and was the major clinical manifestation with the mean baseline body temperature being $37.5 \pm 0.62$. The artemether-lumefantrine combination has been reported as a fast-acting drug with rapid effect on parasite clearance, rapid fever reduction and a longacting drug to prevent recrudescence [33,38-39] contrary to data obtained from Southeast Asian countries such as Thailand and Cambodia including some African countries [40]. Of major concern for ACTs in Africa especially in areas of intense transmission is the sluggish clearance of the parasite thus facilitating the development of resistant strains, necessitating the need for continuous surveillance of its efficacy.

\section{Conclusion}

This study concludes that AL continue to be effective against uncomplicated malaria caused by Plasmodium falciparum in Kenya. We observed high AL efficacy despite the use of this drug for over a decade making it a drug of choice. The drug was well tolerated in this age group and there was no adverse effect during the treatment. However, intensive and regular surveillance of ACT partner drugs needs be conducted to facilitate early detection of resistance to $P$. falciparum to inform policy makers on decisions on malaria treatment.

\section{Limitation of the study.}

The outcome of the study could not be generalized because the small sample size and study population of children at age 6 to 60 months were recruited and only those that completed the study up to day 28 were considered in the analysis. In addition, molecular markers that are implicated in AL resistance were not included.

\section{Abbreviations}
$\mathrm{AL}$ Artemether-lumefantrine
SP Sulfadoxine-pyrimethamine
WHO World Health Organization
ETF Early treatment failure
LCF Late clinical failure 
LPF Late parasitological failure

ACPR Adequate clinical and parasitological response

Pfdhfr Plasmodium falciparum dihydrofolate reductase

Pfdhps Plasmodium falciparum dihydropteroate synthase

IPTp Intermittent and preventive treatment programme

ASAQ Artesunate-amodiaquine

ASSP Artesunate-sulfadoxine-pyrimethamine

DHA/PPQ Dihydroartemisinin-piperaquine

ASMQ Artesunate-mefloquine

PCR Polymerase chain reaction

RFLP Restriction fragment length polymorphism

\section{Declarations}

\section{Acknowledgement}

This study was sponsored by National Council of Science Technology and Innovation (NACOSTI). We would like to acknowledge all members of Chulaimbo Sub County Hospital especially the administration, nurses and laboratory technologists. We appreciate the all the patients and their parents for participating in the study. The malaria team at CBRD, KEMRI for they support during laboratory analysis.

\section{Author contributions}

GK was involved in study design, data collection, and analysis, interpretation of the results and drafting of the manuscript. KT and GO were involved in data collection and manuscript writing, EN FK, DMM were involved in supervision, interpretation of the results and drafting of the manuscript. All authors read and approved the final manuscript.

\section{Funding}

Funding was partly self-sponsored and partly sponsored by the National Council of Science Technology and Innovation (NACOSTI) Ref No. $5^{\text {TH }}$ calls 2013/2014

\section{Availability of data and materials}

The raw data is available on request by the editor of publishing journal 


\section{Ethics approval and consent to participate}

The study was approved by the Scientific Ethics Research Unit of the Kenya Medical Research Institute (SSC Protocol no. 2406) and the consent was obtained from the parents of the participating children

\section{Consent for publication}

Consent was obtained from the mothers that the results can be published provided the names of the children should not appear anywhere.

\section{Competing interest}

The authors declare that they have no competing interests

\section{References}

1. World Health Organization. World malaria report 2019.

2. World Health Organization. Antimicrobial resistance and primary health care: brief. World Health Organization; 2018.

3. Ishengoma DS, Mandara Cl, Francis F, Talundzic E, Lucchi NW, Ngasala B, Kabanywanyi AM, Mahende MK, Kamugisha E, Kavishe RA, Mohamed A. Efficacy and safety of artemetherlumefantrine for the treatment of uncomplicated malaria and prevalence of Pfk13 and Pfmdr1 polymorphisms after a decade of using artemisinin-based combination therapy in mainland Tanzania. Malaria journal. 2019 Dec; 18(1):88.

4. Steketee RW, Campbell CC. Impact of national malaria control scale-up programmes in Africa: magnitude and attribution of effects. Malaria journal. 2010 Dec 1;9(1):299.

5. Hemingway J, Shretta R, Wells TN, Bell D, Djimdé AA, Achee N, Qi G. Tools and strategies for malaria control and elimination: what do we need to achieve a grand convergence in malaria? PLoS biology. 2016 Mar;14(3).

6. Kiarie WC, Wangai L, Agola E, Kimani FT, Hungu C. Chloroquine sensitivity: diminished prevalence of chloroquine-resistant gene marker pfcrt-7613 years after cessation of chloroquine use in Msambweni, Kenya. Malaria journal. 2015 Dec 1;14(1):328.

7. Ndong Ngomo JM, Mawili-Mboumba DP, M’Bondoukwe NP, Nikiéma Ndong Ella R, Bouyou Akotet MK. Increased prevalence of mutant allele Pfdhps $437 \mathrm{G}$ and Pfdhfr triple mutation in Plasmodium falciparum isolates from a rural area of Gabon, three years after the change of malaria treatment policy. Malaria research and treatment. 2016;2016.

8. Okombo J, Kamau AW, Marsh K, Sutherland CJ, Ochola-Oyier LI. Temporal trends in prevalence of Plasmodium falciparum drug resistance alleles over two decades of changing antimalarial policy in coastal Kenya. International Journal for Parasitology: Drugs and Drug Resistance. 2014 Dec 1;4(3):152-63. 
9. World Health Organization. Artemisinin resistance and artemisinin-based combination therapy efficacy: status report. World Health Organization; 2018.

10. World Health Organization. Method for surveillance of antimalarial drug efficacy. 2009, Geneva: World Health Organization.

11. World Health Organization. Global malaria program. Atemisinin resistance and Artemisinin-based combination therapy efficacy.

12. Basco LK, Heseltine E, World Health Organization. Field application of in vitro assays for the sensitivity of human malaria parasites to antimalarial drugs.

13. Witkowski B, Amaratunga C, Khim N, Sreng S, Chim P, Kim S, Lim P, Mao S, Sopha C, Sam B, Anderson JM. Novel phenotypic assays for the detection of artemisinin-resistant Plasmodium falciparum malaria in Cambodia: in-vitro and ex-vivo drug-response studies. The Lancet infectious diseases. 2013 Dec 1;13(12):1043-9.

14. Mwai L, Ochong E, Abdirahman A, Kiara SM, Ward S, Kokwaro G, Sasi P, Marsh K, Borrmann S, Mackinnon M, Nzila A. Chloroquine resistance before and after its withdrawal in Kenya. Malaria journal. 2009 Dec;8(1):106.

15. Nzila AM, Mberu EK, Sulo J, Dayo H, Winstanley PA, Sibley CH, Watkins WM. Towards an understanding of the mechanism of pyrimethamine-sulfadoxine resistance in Plasmodium falciparum: genotyping of dihydrofolate reductase and dihydropteroate synthase of Kenyan parasites. Antimicrobial agents and chemotherapy. 2000 Apr 1;44(4):991-6.

16. Omar SA, Adagu IS, Gump DW, Ndaru NP, Warhurst DC. Plasmodium falciparum in Kenya: high prevalence of drug-resistance-associated polymorphisms in hospital admissions with severe malaria in an epidemic area. Annals of Tropical Medicine \& Parasitology. 2001 Oct 1; 95 (7):661-9.

17. Woodrow CJ, White NJ. The clinical impact of Artemisinin resistance in Southeast Asia and the potential future spread. FEMS Microbiol Rev. 41:1 34-48

18. Shah MM, Krystosik AR, Ndenga BA, Mutuku FM, Caldwell JM, Otuka V, Chebii PK, Maina PW, Jembe Z, Ronga C, Bisanzio D. Malaria smear positivity among Kenyan children peaks at intermediate temperatures as predicted by ecological models. Parasites \& vectors. 2019 Dec;12(1):288.

19. Lwanga SK, Lemeshow S, World Health Organization. Sample size determination in health studies: a practical manual. World Health Organization; 1991.

20. Morris U. Molecular methods in malaria control in the era of pre-elimination. Inst för mikrobiologi, tumör-och cellbiologi/Dept of Microbiology, Tumor and Cell Biology; 2015 Mar 3.

21. World Health Organization. Guidelines for the treatment of malaria. World Health Organization; 2015 Aug 13.

22. Walsh PS, Metzger DA, Higuchi R. Chelex 100 as a medium for simple extraction of DNA for PCRbased typing from forensic materials. Bio Techniques. 1991. 10:4 506-513

23. Warhurst DC, EL KARIEM AW, Miles MA. Simplified preparation of malarial blood samples for polymerase chain reaction. Lancet (British edition). 1991;337(8736):303-4. 
24. Demas A, Oberstaller J, DeBarry J, Lucchi NW, Srinivasamoorthy G, Sumari D, Kabanywanyi AM, Villegas L, Escalante AA, Kachur SP, Barnwell JW. Applied genomics: data mining reveals speciesspecific malaria diagnostic targets more sensitive than $18 \mathrm{~S}$ rRNA. Journal of clinical microbiology. 2011 Jul 1;49(7):2411-8.

25. Menard D, Dondorp A. Antimalarial drug resistance: a threat to malaria elimination. Cold Spring Harbor perspectives in medicine. 2017 Jul 1;7(7):a025619.

26. World Health Organization. Antimicrobial resistance and primary health care: brief. World Health Organization; 2018.

27. Tavul L, Hetzel MW, Teliki A, Walsh D, Kiniboro B, Rare L, Pulford J, Siba PM, Karl S, Makita L, Robinson L. Efficacy of artemether-lumefantrine and dihydroartemisinin-piperaquine for the treatment of uncomplicated malaria in Papua New Guinea. Malaria journal. 2018 Dec;17(1):350.

28. Smith SJ, Kamara AR, Sahr F, Samai M, Swaray AS, Menard D, Warsame M. Efficacy of artemisininbased combination therapies and prevalence of molecular markers associated with artemisinin, piperaquine and sulfadoxine-pyrimethamine resistance in Sierra Leone. Acta tropica. 2018 Sep 1;185:363-70.

29. Mandara Cl, Kavishe RA, Gesase S, Mghamba J, Ngadaya E, Mmbuji P, Mkude S, Mandike R, Njau R, Mohamed A, Lemnge MM. High efficacy of artemether-lumefantrine and dihydroartemisininpiperaquine for the treatment of uncomplicated falciparum malaria in Muheza and Kigoma Districts, Tanzania. Malaria journal. 2018 Dec;17(1):261.

30. Kakolwa MA, Mahende MK, Ishengoma DS, Mandara Cl, Ngasala B, Kamugisha E, Kataraihya JB, Mandike R, Mkude S, Chacky F, Njau R. Efficacy and safety of artemisinin-based combination therapy, and molecular markers for artemisinin and piperaquine resistance in Mainland Tanzania. Malaria journal. 2018 Dec 1;17(1):369.

31. World Health Organization. World malaria report 2015. World Health Organization; 2016 Jan 30.

32. Djimde AA, Makanga M, Kuhen $K$, Hamed $K$. The emerging threat of artemisinin resistance in malaria: focus on artemether-lumefantrine. Expert review of anti-infective therapy. $2015 \mathrm{Aug}$ 3;13(8):1031-45.

33. Teklemariam M, Assefa A, Moges Kassa HM, Mamo H. Therapeutic efficacy of artemetherlumefantrine against uncomplicated Plasmodium falciparum malaria in a high-transmission area in northwest Ethiopia. PLoS One. 2017;12(4).

34. Cui L, Mharakurwa S, Ndiaye D, Rathod PK, Rosenthal PJ. Antimalarial drug resistance: literature review and activities and findings of the ICEMR network. The American journal of tropical medicine and hygiene. 2015 Sep 2;93(3_Suppl):57-68.

35. Mekonnen SK, Medhin G, Berhe N, Clouse RM, Aseffa A. Efficacy of artemether-lumefantrine therapy for the treatment of uncomplicated Plasmodium falciparum malaria in Southwestern Ethiopia. Malaria journal. 2015 Dec;14(1):317.

36. Omondi P, Burugu M, Matoke-Muhia D, Too E, Nambati EA, Chege W, Musyoka KB, Thiongo K, Otinga M, Muregi F, Kimani F. Gametocyte clearance in children, from western Kenya, with uncomplicated 
Plasmodium falciparum malaria after artemether-lumefantrine or dihydroartemisinin-piperaquine treatment. Malaria Journal. 2019 Dec 1;18(1):398.

37. Ouji M, Augereau JM, Paloque L, Benoit-Vical F. Plasmodium falciparum resistance to artemisininbased combination therapies: A sword of Damocles in the path toward malaria elimination. Parasite. 2018;25.

38. Färnert A, Ursing J, Tolfvenstam T, Rono J, Karlsson L, Sparrelid E, Lindegårdh N. Artemetherlumefantrine treatment failure despite adequate lumefantrine day 7 concentration in a traveller with Plasmodium falciparum malaria after returning from Tanzania. Malaria journal. 2012 Dec 1;11(1):176.

39. Nega D, Assefa A, Mohamed H, Solomon H, Woyessa A, Assefa Y, Kebede A, Kassa M. Therapeutic efficacy of artemether-lumefantrine (Coartem ${ }^{\circledR}$ ) in treating uncomplicated P. falciparum malaria in Metehara, Eastern Ethiopia: regulatory clinical study. PLoS One. 2016;11(4).

40. Byakika-Kibwika P, Nyakato P, Lamorde M, Kiragga AN. Assessment of parasite clearance following treatment of severe malaria with intravenous artesunate in Ugandan children enrolled in a randomized controlled clinical trial. Malaria journal. 2018 Dec 1;17(1):400.

\section{Figures}




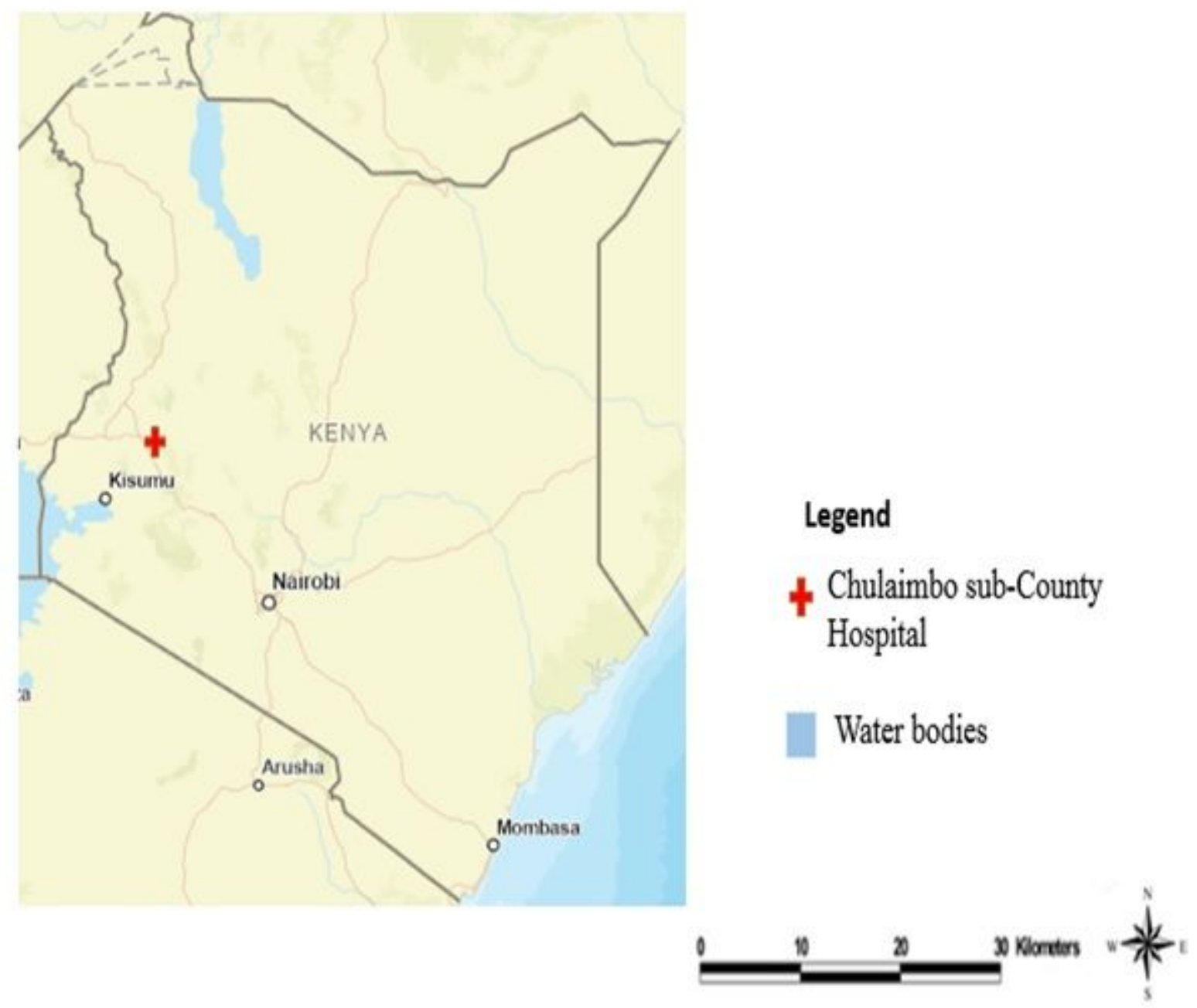

Figure 1

A map showing Chulaimbo sub-County Hospital, Kisumu County, Western Kenya 


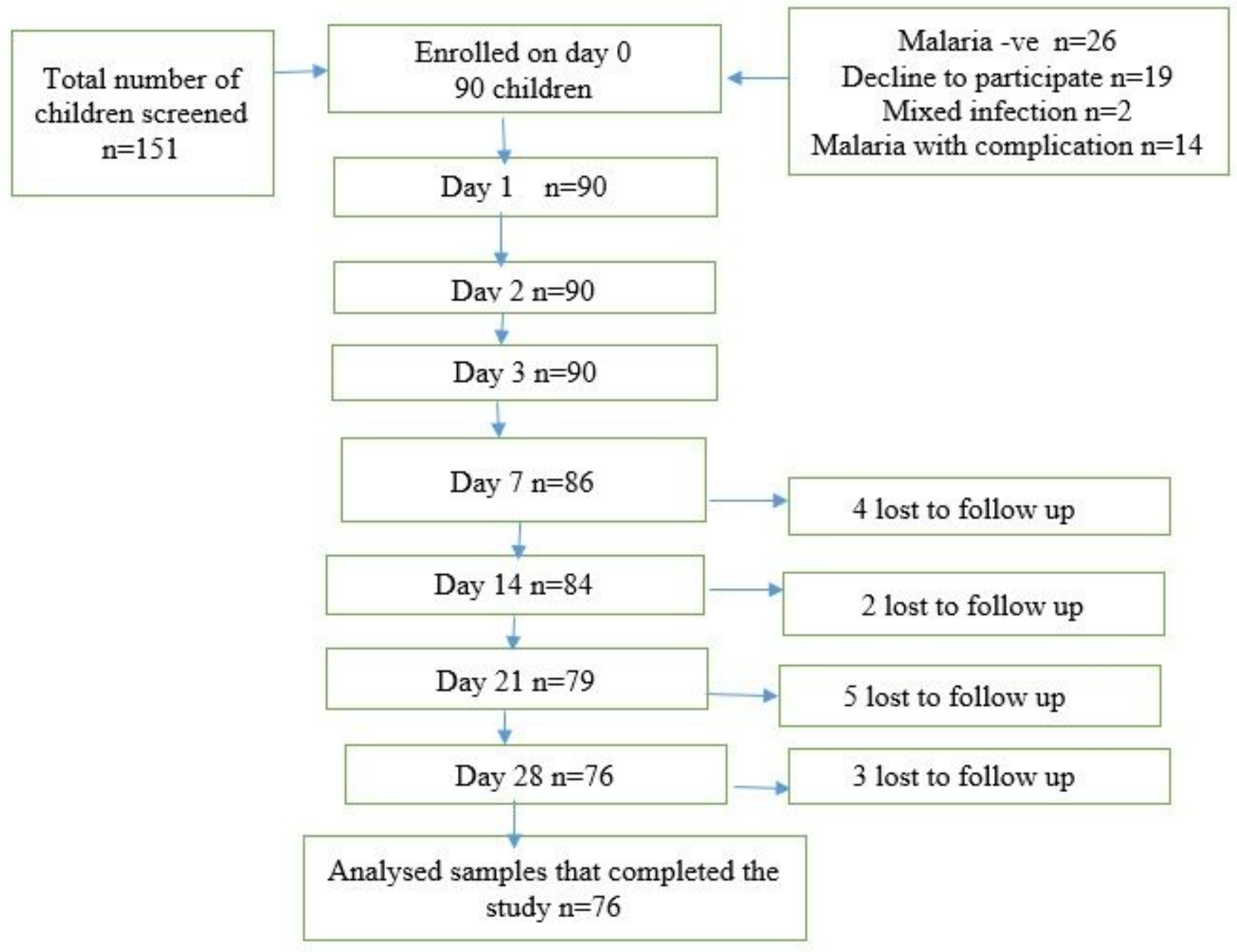

Figure 2

Therapeutic efficacy study showing the flow of patients during the screening, enrolment and follow up.

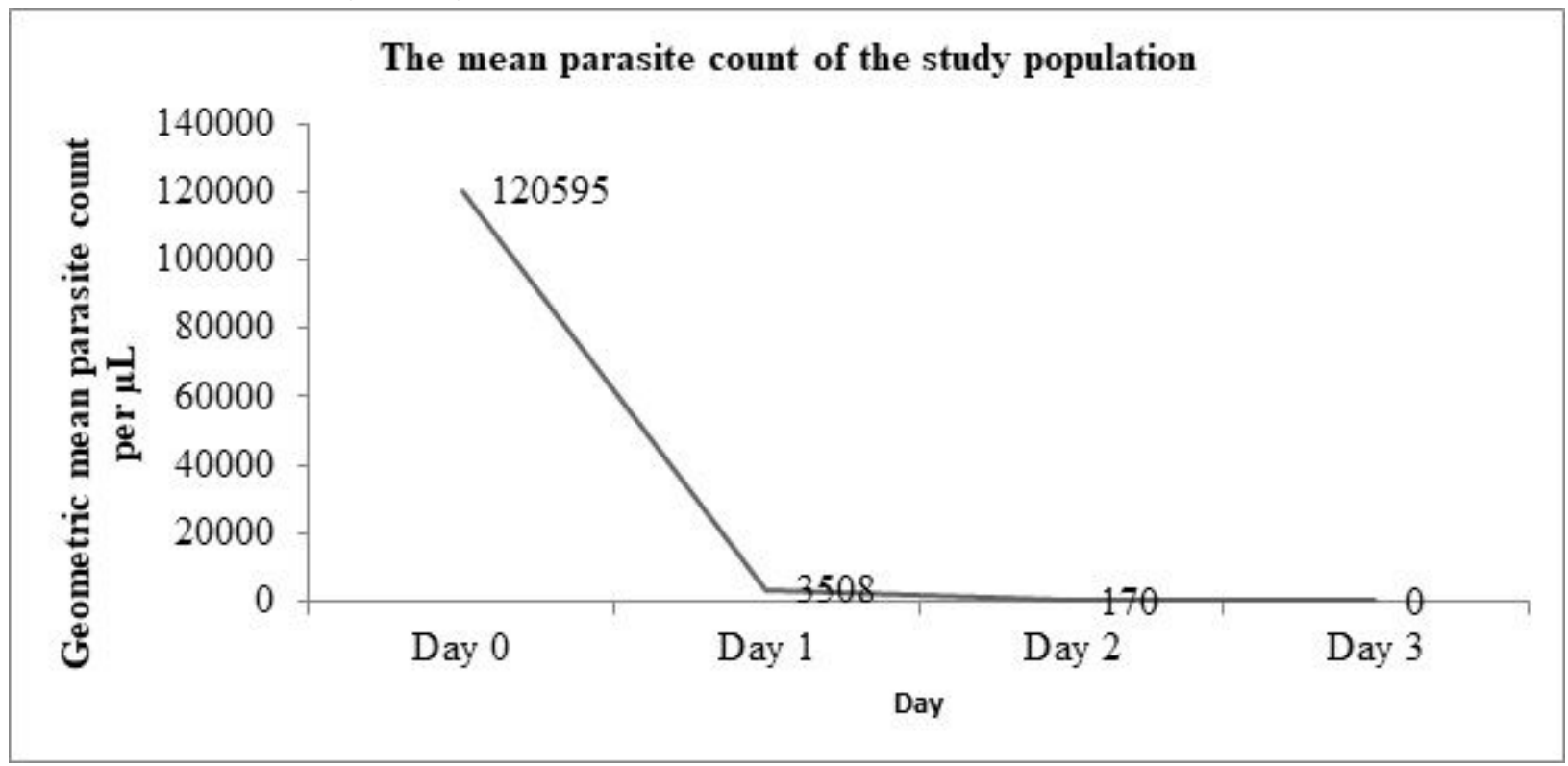

Figure 3 
Presentation of mean parasite density on the first three follow-up days

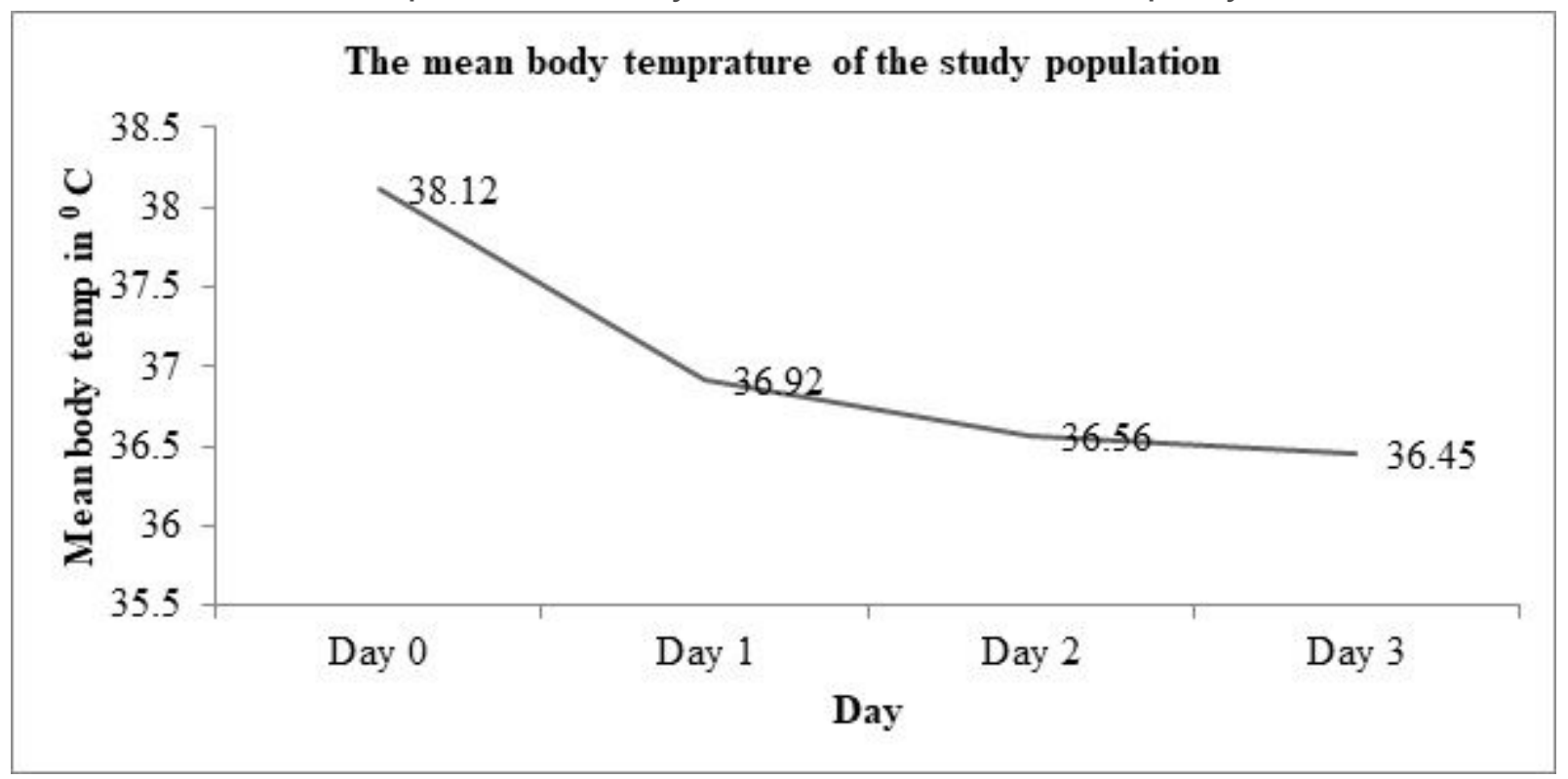

Figure 4

Presentation of mean body temperature on the first three follow-up days

Kaplan - Meier Analysis

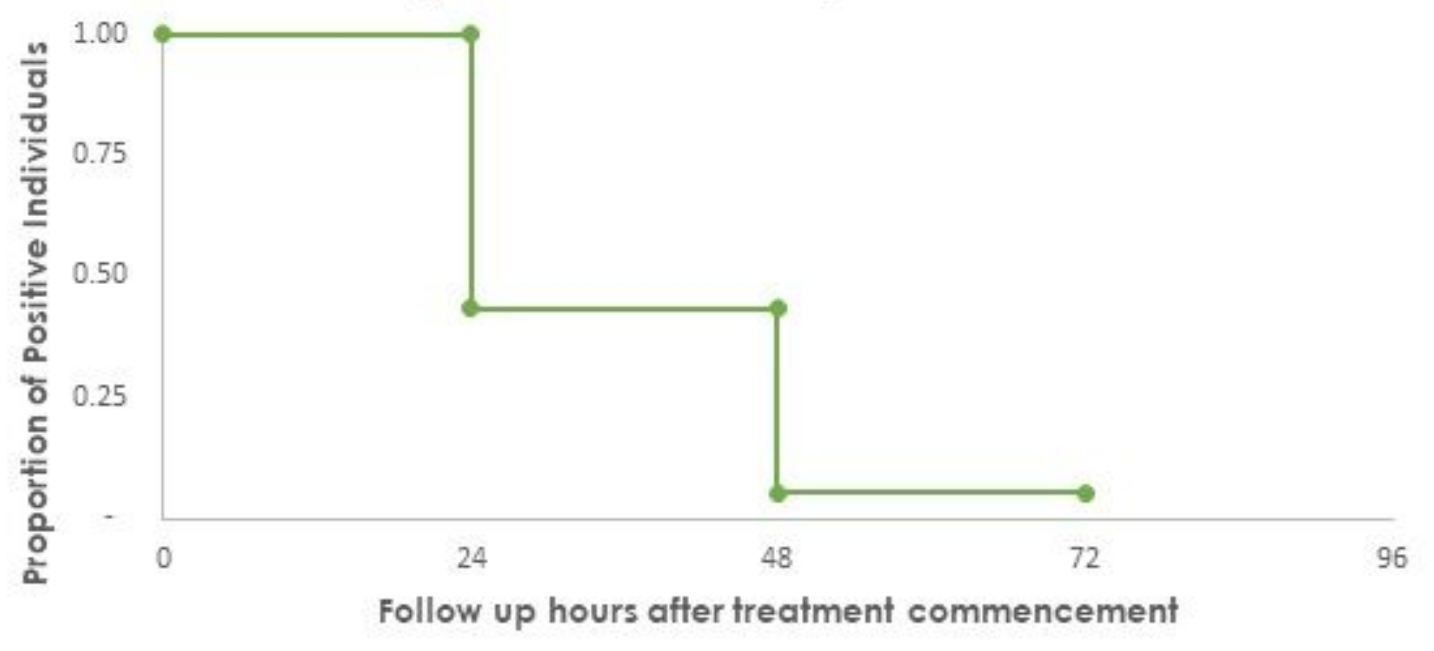

\section{Figure 5}

Kaplan-Meier plot of time versus the individual whose parasites were cleared after treatment. The proportion of Plasmodium falciparum positive children are shown on $\mathrm{Y}$-axis while time of disappearance of the parasites is shown on the X-axis 